\title{
On the effect of crystallographic orientation on ductile material removal in silicon
}

\author{
Brian P. O'Connor ${ }^{\mathrm{a}}$, Eric R. Marsh ${ }^{\mathrm{b}, *}$, Jeremiah A. Couey ${ }^{\mathrm{b}}$ \\ a Aerotech, Inc., 101 Zeta Drive, Pittsburgh, PA 15238, USA \\ b The Pennsylvania State University, 21 Reber Building, University Park, PA 16802, USA
}

Received 9 October 2003; received in revised form 10 March 2004; accepted 19 May 2004

Available online 28 July 2004

\begin{abstract}
In this work the critical chip thickness for ductile regime machining of monocrystalline, electronic-grade silicon is measured as a function of crystallographic orientation on the $(001)$ cubic face. A single-point diamond flycutting setup allows sub-micrometer, non-overlapping cuts in any direction while minimizing tool track length and sensitivity to workpiece flatness. Cutting tests are performed using chemically faceted, $-45^{\circ}$ rake angle diamond tools at cutting speeds of 1400 and $5600 \mathrm{~mm} / \mathrm{s}$. Inspection of the machined silicon workpiece using optical microscopy allows calculation of the critical chip thickness as a function of crystallographic orientation for different cutting conditions and workpiece orientations. Results show that the critical chip thickness in silicon for ductile material removal reaches a maximum of $120 \mathrm{~nm}$ in the [1 00 ] direction and a minimum of $40 \mathrm{~nm}$ in the [1 10 ] direction. These results agree with the more qualitative results of many previous efforts.
\end{abstract}

(C) 2004 Elsevier Inc. All rights reserved.

Keywords: Diamond turning; Silicon; Critical chip thickness; Crystallographic orientation

\section{Introduction}

Hard, brittle materials such as silicon are commonly used in infrared optics and semiconductor-based products; however, machining of these low fracture toughness materials is complicated by stringent requirements on form accuracy, surface finish and subsurface damage. Grinding and polishing are used to meet these challenges, but are widely recognized to be decidedly slow and costly in most applications. As a result, there is a continuing motivation to use diamond turning to eliminate at least some of the steps. The literature includes many works devoted to making cost effective and high quality silicon components with single point diamond tools.

Silicon is nominally diamond turnable based upon its chemical composition, but it causes significant tool wear resulting in a steady deterioration of surface finish and an increase of subsurface damage [1]. Furthermore, relatively small depths of cut and feed rates are required for material removal in the ductile regime.

The literature documents a comprehensive exploration of the properties and behavior of silicon and crystallographically similar diamond. Both materials show directionally de-

\footnotetext{
${ }^{*}$ Corresponding author. Tel.: +1-814-865-5242; fax: +1-814-865-8682.

E-mail address: emarsh@psu.edu (E.R. Marsh).
}

pendent behavior including preferred orientations for best results with various material removal processes [2-5]. For example, Wilks and Wilks showed that diamond polishing rates vary when working the crystal in different directions [6]. Furthermore, silicon turned with single point tools can show radial spokes of damage in the directions predicted to be the most difficult by Wilks and Wilks. Blackley and Scattergod explained the orientation dependent machining damage in silicon by examining the variation in resolved tensile stress on the $\left\{\begin{array}{lll}1 & 1 & 1\end{array}\right\}$ slip planes [7]. Shibata et. al. observed the same pitting damage when turning silicon and qualitatively explained the direction-dependent damage effects by the use of a slip model [8]. Recent studies have shown that silicon transforms to a metallic phase ( $\beta$-tin) under the compressive loading of the cutting tool $[9,10]$. If the depth of cut is less than the depth of the transformed metallic phase, the material removal process will behave as expected for a ductile material. However, if the depth of cut is too aggressive and exceeds the dimension of the metallic phase, then the material will be removed by brittle fracture [11].

The crystal structure of silicon (diamond cubic at room temperature) is shown in Fig. 1. The structure of the crystal lattice and atomic arrangement are extremely important in understanding the anisotropy of silicon and affects its mechanical, electrical, chemical and optical properties. 


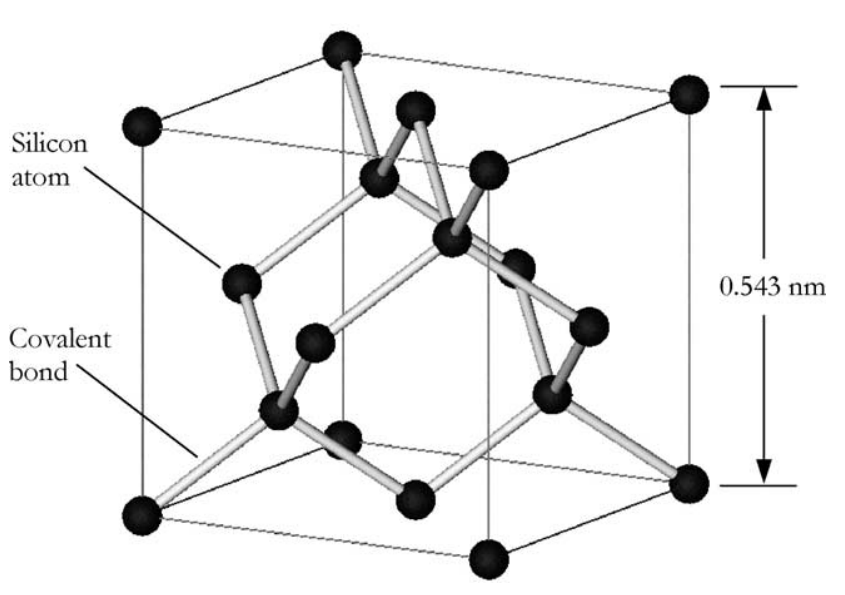

Fig. 1. Diamond cubic crystal structure of silicon.

The mechanical anisotropy is especially influential on the orientation-dependent results obtained when machining silicon. The silicon crystal atomic structure viewed from the [1 000$]$ direction is shown in Fig. 2 with the darkened atoms representing silicon atoms on a single atomic plane.

One example of the anisotropy in the mechanical properties of silicon is found in the elastic modulus variation. The elastic modulus for a cubic crystal can be represented as

$E^{-1}=s_{11}-2\left(s_{11}-s_{12}-\frac{1}{2} s_{44}\right)\left(l_{1}^{2} l_{2}^{2}+l_{2}^{2} l_{3}^{2}+l_{1}^{2} l_{3}^{2}\right)$

where $s_{11}, s_{12}$, and $s_{44}$ are the elastic compliance constants and $l_{1}, l_{2}$, and $l_{3}$ are the direction cosines relative to the cubic crystal axes [12]. Fig. 3 shows the elastic modulus of silicon as a function of crystal orientation. For a purely isotropic material the elastic modulus in three-dimensional space is spherical.

The variation of the elastic modulus on individual crystal planes is found by taking slices of the three-dimensional surface through the origin of the crystal axes. Fig. 4 shows the elastic modulus variation on the cubic face of monocrystalline silicon as calculated using Eq. (1). On the cubic crystal

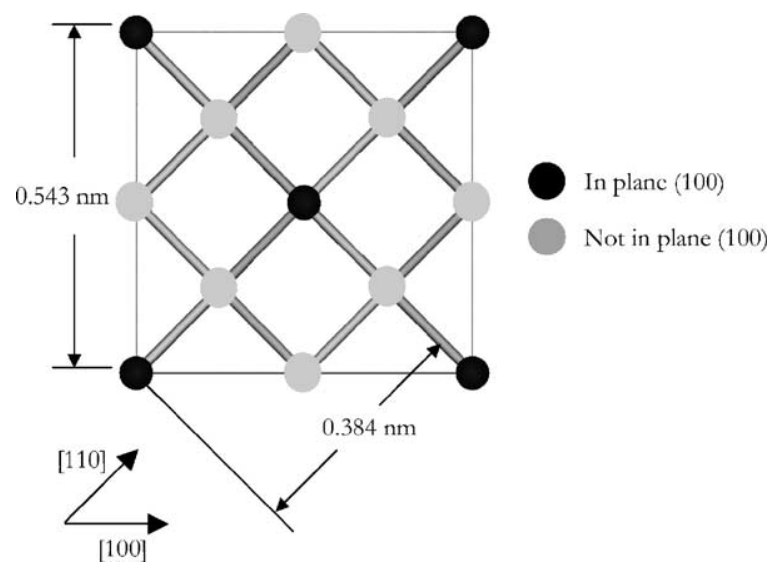

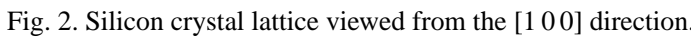

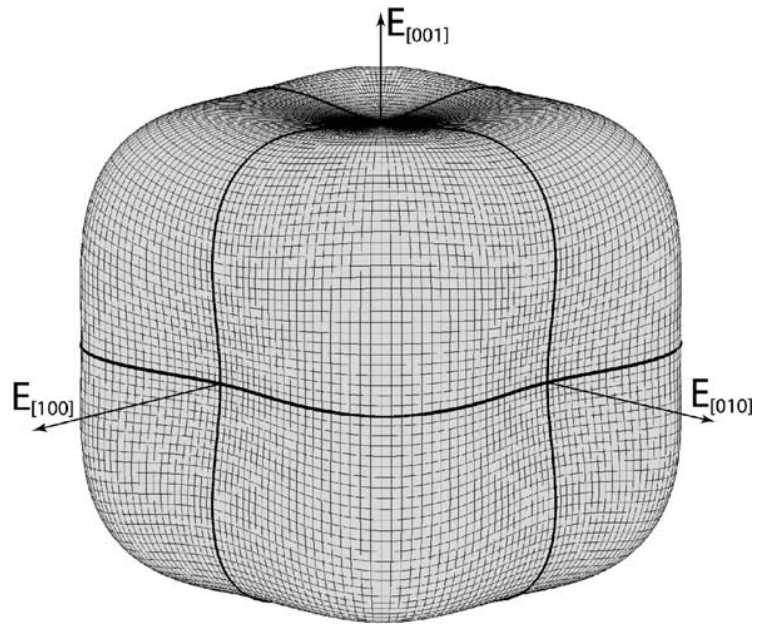

Fig. 3. Elastic modulus anisotropy of silicon.

plane, a 4-lobed pattern emerges in the elastic modulus variation. This variation mirrors the atomic structure on the $\left(\begin{array}{lll}0 & 0 & 1\end{array}\right)$ crystal face shown in Fig. 2, and a pattern repeating every $90^{\circ}$ is evident. As expected, the direction with the highest atomic density yields the highest elastic modulus.

Since silicon is macroscopically brittle, other material properties such as fracture toughness and hardness can influence material removal as suggested by Bifano et al. [3]. The nature of hardness anisotropy in crystals is governed by the atomic structure of the material and the primary slip systems that aid dislocation motion during indentation [13]. It has been found that the crystallographic directions corresponding to minimum values of the effective resolved shear stress are those of maximum hardness. Brookes and Burnand [14] give the effective resolved shear stress in a material as

$\tau_{\mathrm{e}}=\frac{F}{2 A}(\cos \psi+\sin \gamma) \cos \alpha \cos \lambda$

where $F$ is the applied load, $A$ is the projected area supporting the load, $\psi$ is the angle between each face of the indenter and the axis of rotation for the slip system, $\gamma$ is the angle between each face of the indenter and the slip direction, $\alpha$ is the angle between the axis of the applied load and the normal vector to the slip plane, and $\lambda$ is the angle between the axis of the applied load and the slip direction.

The recent work in nanoindentation of silicon has not yet explored hardness variations as a function of indenter orientation $[9,10,15]$. However, there are published Knoop hardness values for single crystal diamond, as shown in Table 1.

Fracture toughness is also important in understanding the machining process of silicon. For silicon, the fracture tough-

Table 1

Knoop indentation hardness with $1 \mathrm{~kg}$ load in diamond (after [11])

\begin{tabular}{lll}
\hline Crystal plane & Direction & Hardness $\left(\mathrm{kg} / \mathrm{mm}^{2}\right)$ \\
\hline$\left(\begin{array}{lll}0 & 0 & 1\end{array}\right)$ & {$\left[\begin{array}{lll}1 & 1 & 0\end{array}\right]$} & 6900 \\
$\left(\begin{array}{lll}0 & 0 & 1\end{array}\right)$ & {$\left[\begin{array}{lll}1 & 0 & 0\end{array}\right]$} & 9600
\end{tabular}




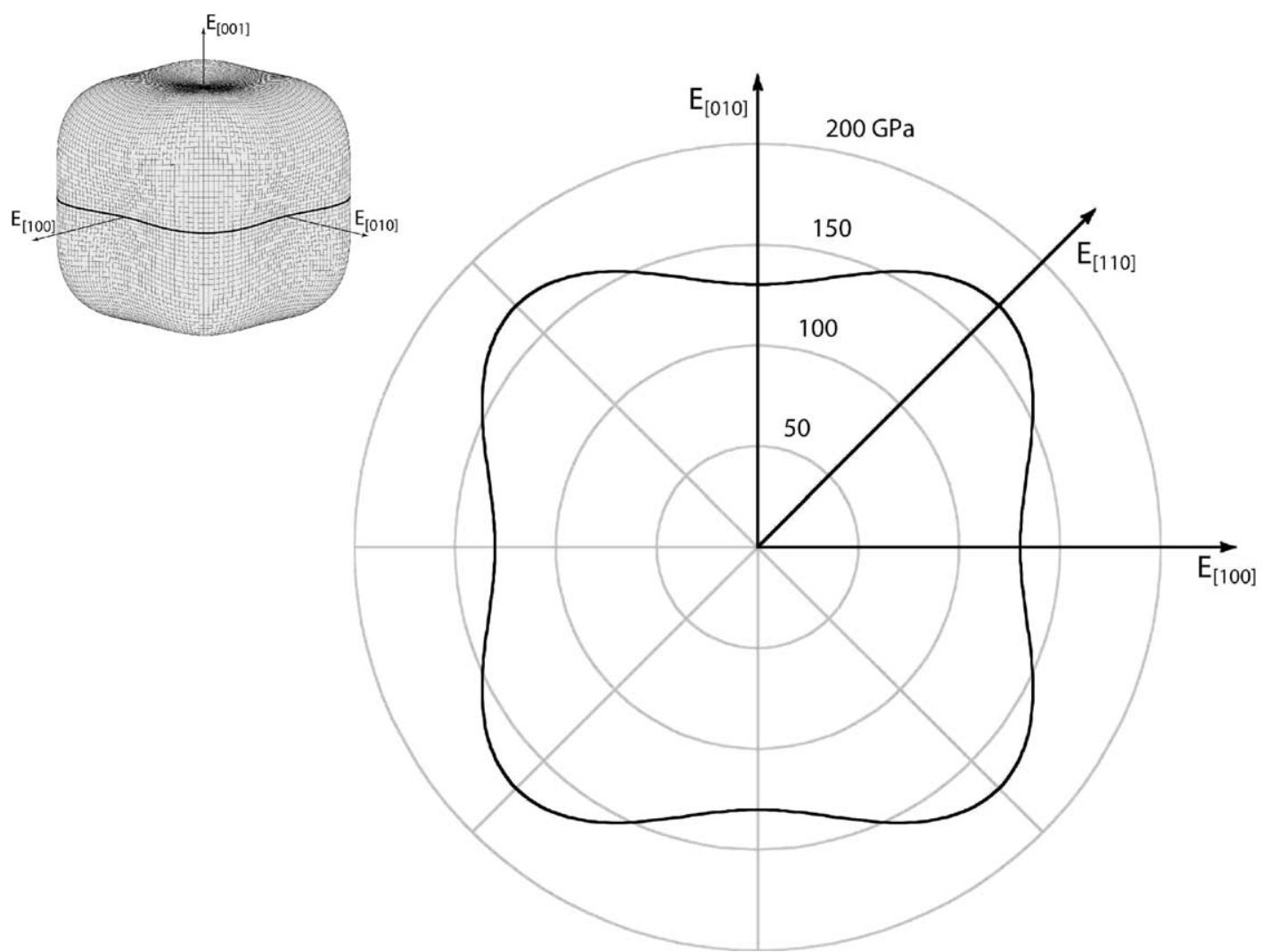

Fig. 4. Variation of the elastic modulus around the (l 001$)$ crystal plane of silicon. The elastic modulii in the [ 100$]$ and $\left[\begin{array}{lll}1 & 0 & 0\end{array}\right]$ directions are 130 and $170 \mathrm{GPa}$, respectively.

ness is found to vary with the crystal plane orientation. Chen and Leipold give the values of fracture toughness in certain crystal planes [16] as shown in Table 2. Although no information is available about the fracture toughness for different crystallographic orientations within a given plane, the tabulated values indicate the amount of variation within the crystal.

The ductile regime machining behavior of silicon varies as a function of crystallographic orientation with trends similar to the variation in material properties. To further explore how these material properties affect the machining behavior we ran an experiment using two spindles (a flycutter spindle and a work spindle) to make interrupted, non-overlapping cuts over the entire crystal face. The advantage of using a two-spindle flycutting approach is that tool track length is minimized to reduce or eliminate tool wear over the course of a single test. By making non-overlapping cuts, there is no damage from previous tool passes in the machined workpiece. As a result, the grooves cut by each pass of the diamond tool provide

Table 2

Fracture toughness variation in silicon after Chen and Leipold [16]

\begin{tabular}{ll}
\hline Crystal plane & Fracture toughness $\left(\mathrm{MPa} \mathrm{m}^{1 / 2}\right)$ \\
\hline$\left(\begin{array}{lll}1 & 0 & 0\end{array}\right)$ & 0.95 \\
$\left(\begin{array}{lll}1 & 1 & 0\end{array}\right)$ & 0.90 \\
$\left(\begin{array}{lll}1 & 1 & 1\end{array}\right)$ & 0.82 \\
\hline
\end{tabular}

information about the ductile to brittle transition (critical chip thickness) and machining force in a specific crystallographic direction.

It is important to note that this cutting geometry is fundamentally different from conventional turning. In turning, some amount of damage may be left behind as the tool passes, but as long as the damage does not propagate below the depth of cut, the finished surface will appear to be cut in the ductile regime. In the interrupted flycutting geometry, the cuts do not overlap, so any brittle fracture is detectable. As a result of this distinction, the critical chip thicknesses reported in this work may prove to be somewhat conservative if applied to a turning process.

All tests were performed on the $(001)$ cubic face. The experimental setup is described along with details on the calculation of the critical chip thickness using reflectedlight optical microscopy. An uncertainty analysis is then presented that estimates the effects of the major error components involved in the measurement. Finally, the crystallographic-dependence of the critical chip thickness and sample force data are presented.

\section{Experimental setup}

The silicon flycutting tests were performed on a hydrostatic diamond turning lathe (Moore Nanotechnology Sys- 


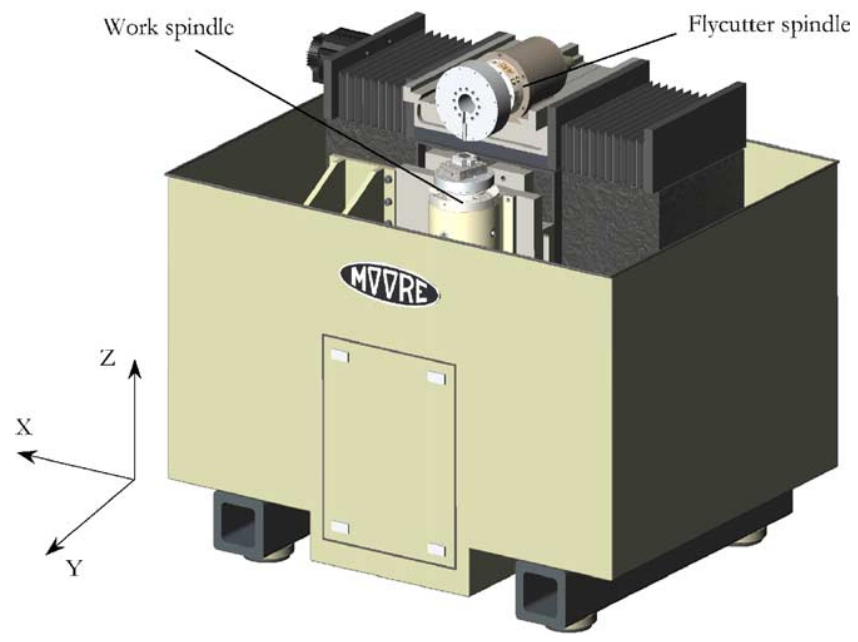

Fig. 5. Experimental setup for silicon flycutting.

tems $150 \mathrm{AG}$ ) with a programmable resolution of $10 \mathrm{~nm}$ in $\mathrm{X}$ and $\mathrm{Z}$ and 1.6 arc-seconds on the $\mathrm{C}$ axis. A solid model of the machine and its two air bearing spindles is shown in Fig. 5.

A three-axis dynamometer (Kistler MiniDyn 9256A2), an acoustic emission sensor (Kistler 8152B2), and a custom workpiece chuck are mounted on the vertical $\mathrm{C}$-axis work spindle (Professional Instruments Twin-Mount). The aluminum workpiece chuck is diamond-turned in assembly to achieve a mounting surface flatness of better than $0.2 \mu \mathrm{m}$. The acoustic emission sensor is mounted to the chuck and is used with an oscilloscope to detect tool-workpiece contact and to monitor the cutting process real-time. Fig. 6 shows a picture of the work spindle and instrumentation layout.
The first resonance of the dynamometer, with the chuck and workpiece, is $3600 \mathrm{~Hz}$. Although the force data is ana$\log$ anti-alias filtered and then sampled at $51.2 \mathrm{kHz}$, a digital lowpass filter is applied in post-processing with a cutoff of $1000 \mathrm{~Hz}$ to remove the spectral components outside the linear response of the instrument. This raises an interesting issue because the spinning diamond tool is only in contact with the workpiece for a few milli-seconds, even at relatively low cutting speeds. At higher spindle speeds the duration of cutting exceeds the bandwidth of the dynamometer. Therefore, the measured force is useful only at a qualitative level because the cutting dynamics are too fast to accurately resolve the different regimes of material response (initial contact with elastic recovery, ductile regime cutting, transition to brittle fracture, all brittle).

Fig. 7 shows a close-up view of the flycutter air bearing spindle (Professional Instruments AC Foot/Flange), flycutter head, and diamond tool. A capacitance probe (Lion Precision DMT-10 C1-C) targets the back of the flycutter and triggers the data acquisition for the force measurement. By selectively triggering the data acquisition, the data file size can be kept manageable by capturing the force only when the tool is cutting.

It is well known in machining brittle materials that a large negative rake angle is beneficial due to its mitigating effects on fracture $[2-5,7,8]$. The synthetic, monocrystalline diamond tools used in the flycutting experiments are $-45^{\circ}$ rake angle, $-10^{\circ}$ clearance angle, and $1.5 \mathrm{~mm}$ nose radius (Edge Technologies, Inc). The tool edge radius, which may be measured in a low voltage SEM (without a graphite or gold vacuum deposition coating) or by AFM, is less than $50 \mathrm{~nm}$ [17]. The tools are chemically faceted on the rake and nose facets

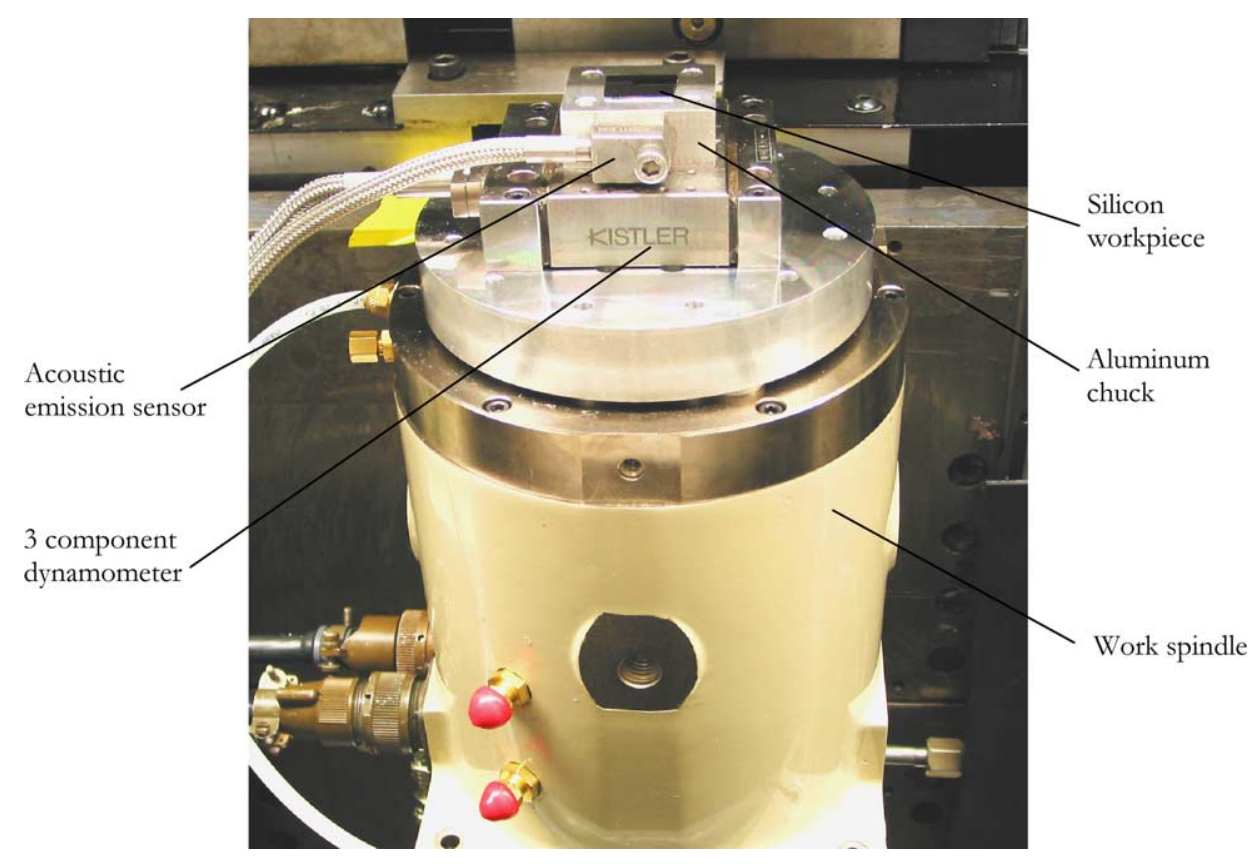

Fig. 6. The work spindle with dynamometer, AE sensor, chuck, and silicon workpiece. 


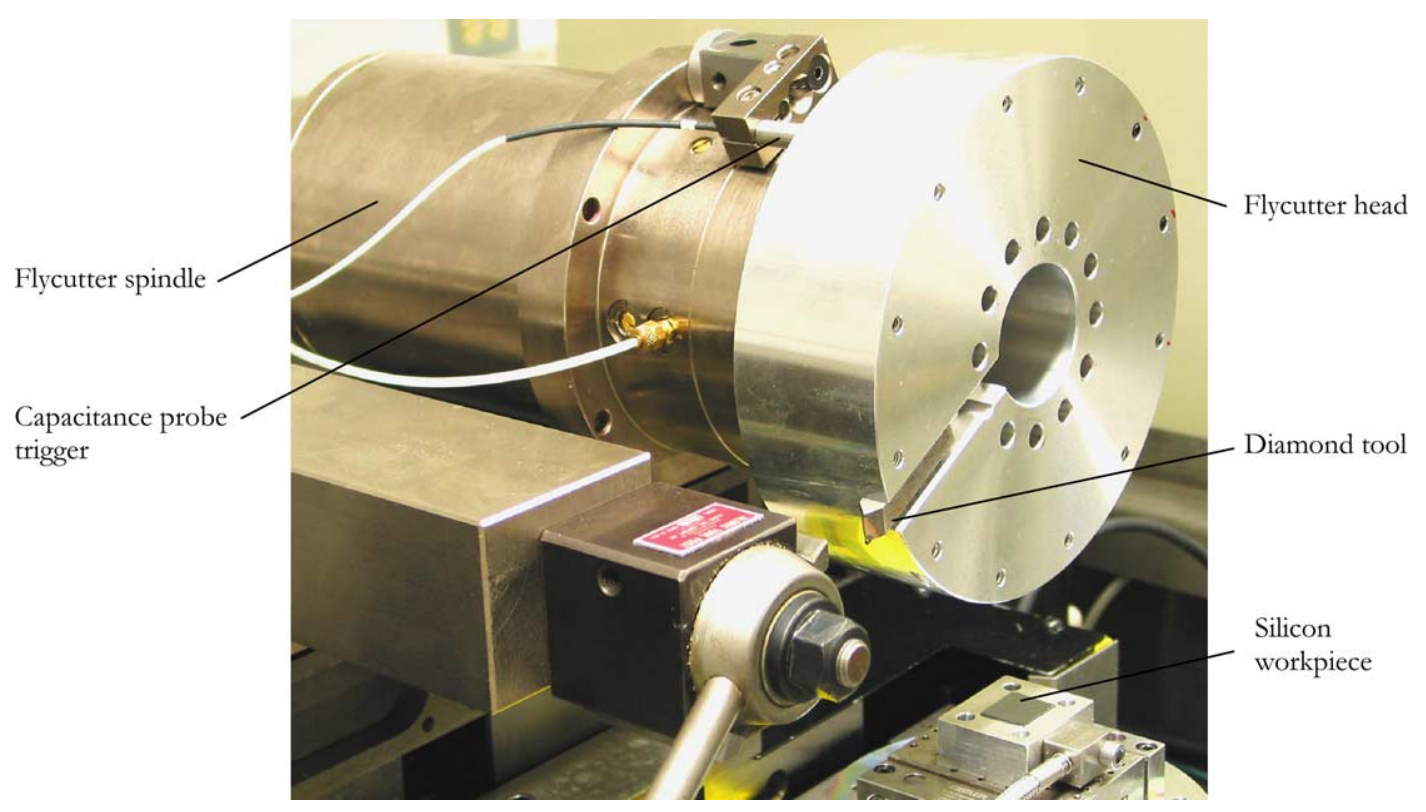

Fig. 7. The flycutter spindle, diamond tool, and workpiece.

to remove surface and subsurface damage from the tool edge. No coolant was used during testing.

The experimental setup allows for small, non-overlapping cuts to be made in any direction on a given plane, in this case the cubic plane. Brinksmeier et al. used a similar setup in work exploring the ductile/brittle transition with silicon [18]. Because of the relatively high diamond tool wear rate in silicon, we added a second axis of rotation to the Brinksmeier configuration to conveniently test many crystal orientations with the lowest possible track length on the single point tool. Fig. 8 illustrates the geometry and kinematics of the machine layout and tool/workpiece interaction during cutting.
The tool path is an arc so the chip thickness varies predictably within each individual cut. This allows the critical chip thickness of the ductile to brittle transition to be calculated from visually identifiable features left behind in the surface of the elliptical groove.

Since the axis of rotation of the flycutter and the axis of rotation of the work spindle are offset by a distance $e$, the cuts are made in a circular pattern around the crystal face. The ratio of flycutter spindle speed to work spindle speed is chosen such that a cut is made every $3^{\circ}$. During the course of each test, the work spindle is rotated $350^{\circ}$ so that defined starting and stopping fiducial marks remain on the machined

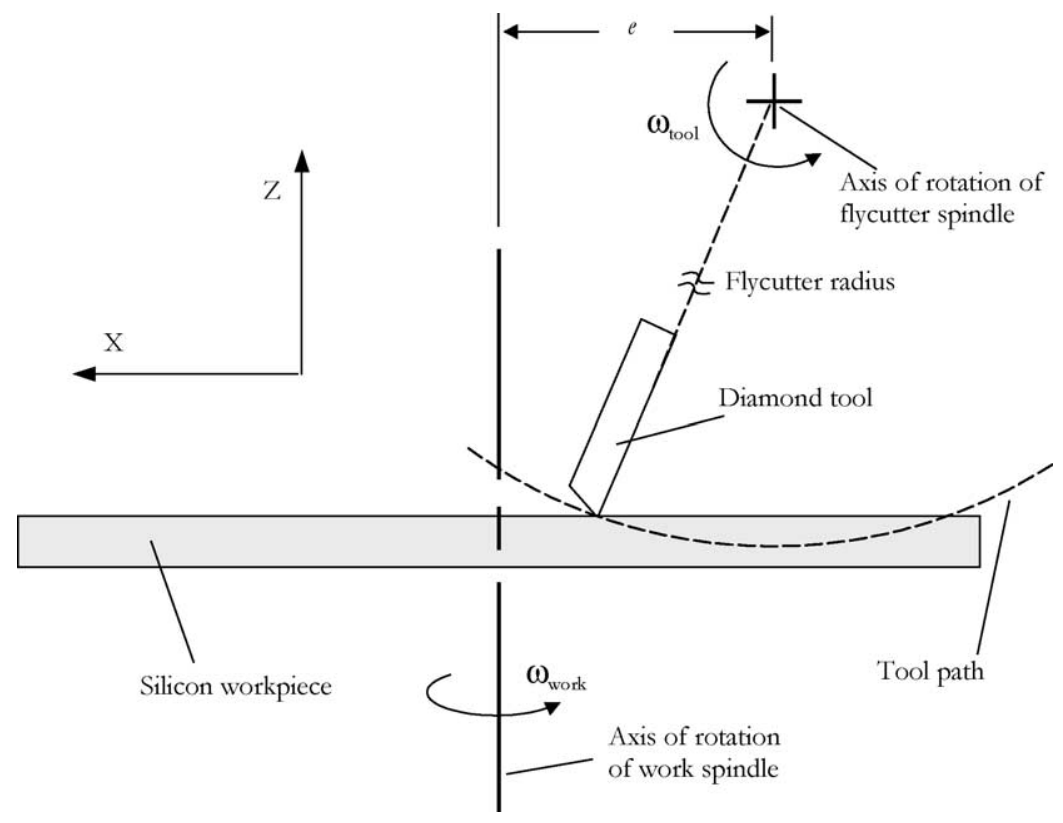

Fig. 8. Schematic of the flycutter and workpiece geometry. 


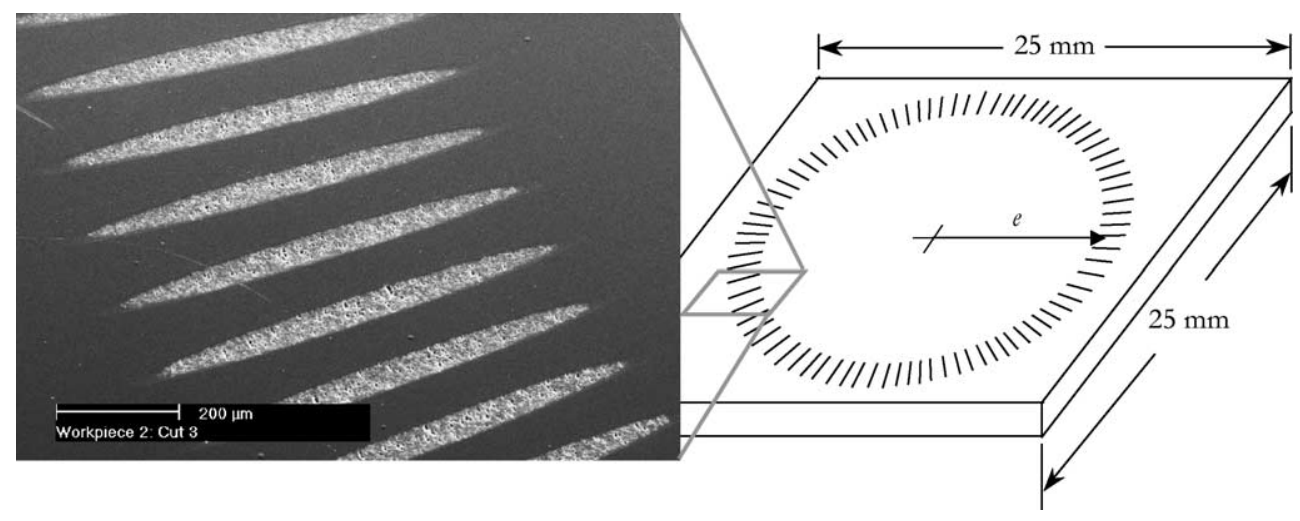

Fig. 9. Example of cuts made on the ( 001$)$ crystal face of monocrystalline silicon.

workpiece and can be later correlated to specific crystallographic directions. Fig. 9 shows an example of a machined workpiece.

This geometry reduces the total tool track length to less than $50 \mathrm{~mm}$ and minimizes the effect of tool wear during the test. In addition, the flycutting tests are insensitive to workpiece flatness because the groove left behind in each individual cut leaves an accurate record of depth of cut as well as the ductile to brittle transition.

\section{Workpiece metrology}

After some exploration using SEM and AFM, the workpieces were found to be best measured using differential interference contrast (Nomarski) optical microscopy to measure each cut on the $\left(\begin{array}{lll}0 & 0 & 1\end{array}\right)$ crystal face. For the purpose of this analysis, the critical chip thickness is defined as the depth of cut where a noticeable change in surface topography starts to occur as the tool cuts deeper into the silicon on its circular path. Fig. 10 shows an individual cut made in a silicon workpiece.

With the knowledge of the flycutter radius $R$ and by measuring the length of the cut $L_{1}$ and the lengths from the beginning and end of the cut to the start of the damaged region, $d_{1}$ and $d_{2}$, respectively, the critical chip thickness $t_{\mathrm{c}}$ is calculated:

$t_{\mathrm{c}}=\frac{L_{1}^{2}-L_{2}^{2}}{8 R}$ where $L_{2}=L_{1}-d_{1}-d_{2}$. Substituting the expression for $L_{2}$ into Eq. (3) yields an expression for critical chip thickness based on the measured quantities $L_{1}, d_{1}, d_{2}$, and $R$.

$t_{\mathrm{c}}=\frac{2\left(L_{1} d_{1}+L_{1} d_{2}-d_{1} d_{2}\right)-d_{1}^{2}-d_{2}^{2}}{8 R}$

A reflected-light optical microscope (Olympus BX60 Nomarski) with a high-resolution digital camera is used to capture images of each cut with $20 \times$ and $50 \times$ objectives. A silicon artifact with $100 \mu \mathrm{m}$ wide etched lines is used as the calibration standard for each magnification. The digital images are imported into a MATLAB ${ }^{\circledR}$ script file and the relevant dimensions of the cut $\left(L_{1}, d_{1}\right.$, and $\left.d_{2}\right)$ are measured to calculate the critical chip thickness using Eq. (4). This process is repeated for each groove in the workpiece.

\subsection{Uncertainty analysis}

This section describes an analysis to estimate the uncertainty in the calculation of critical chip thickness [19]. For a function $f$ of $N$ uncorrelated input estimates $x_{i}$, the variance of $f$ is:

$u^{2}(f)=\sum_{i=1}^{N} u_{i}^{2}(f)=\sum_{i=1}^{N}\left(\frac{\partial f}{\partial x_{i}}\right)^{2} u^{2}\left(x_{i}\right)$

where $u^{2}\left(x_{i}\right)$ is the variance of the input estimate $x_{i}$. The combined standard uncertainty of the function $f$ is $u(f)$. The variance of critical chip thickness $t_{\mathrm{c}}$ is found by using Eqs. (4) and (5).

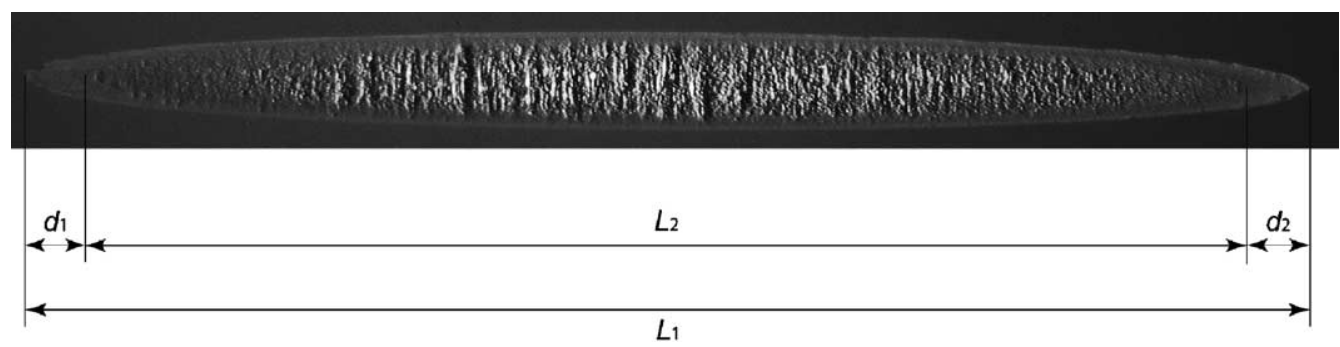

Fig. 10. The geometry of an individual cut made in a silicon workpiece. 
Table 3

Uncertainty analysis for the critical chip thickness calculation based on nominal values of $L_{1}=1.0 \mathrm{~mm}, R=110 \mathrm{~mm}, d_{1}=d_{2}=25 \mu \mathrm{m}$

\begin{tabular}{lccc}
\hline Source of uncertainty & $\begin{array}{l}\text { Measurement } \\
\text { interval }(\mu \mathrm{m})\end{array}$ & $\begin{array}{l}\text { Standard uncertainty } \\
u_{i}\left(t_{\mathrm{c}}\right)(\mu \mathrm{m})\end{array}$ & Sensitivity \\
\hline Measurement of $L_{1}$ & 7.0 & $4.0(\mathrm{~B})$ & $1.1 \times 10^{-4}$ \\
Measurement of $d_{1}$ & 2.8 & $1.6(\mathrm{~B})$ & $2.2 \times 10^{-3}$ \\
Measurement of $d_{2}$ & 2.8 & $1.6(\mathrm{~B})$ & $2.2 \times 10^{-3}$ \\
Measurement of $R$ & 250 & & $-1.0 \times 10^{-6}$ \\
Combined standard uncertainty $u\left(t_{\mathrm{c}}\right)$ & & & $5.0 \mathrm{~nm}$ \\
Expanded uncertainty $(k=2)$ & & & $10.0 \mathrm{~nm}$ \\
\hline
\end{tabular}

$$
\begin{aligned}
u^{2}\left(t_{\mathrm{c}}\right)= & \left(\frac{\partial t_{\mathrm{c}}}{\partial L_{1}}\right)^{2} u^{2}\left(L_{1}\right)+\left(\frac{\partial t_{\mathrm{c}}}{\partial d_{1}}\right)^{2} u^{2}\left(d_{1}\right) \\
& +\left(\frac{\partial t_{\mathrm{c}}}{\partial d_{2}}\right)^{2} u^{2}\left(d_{2}\right)+\left(\frac{\partial t_{\mathrm{c}}}{\partial R}\right)^{2} u^{2}(R)
\end{aligned}
$$

For the purpose of estimating the uncertainty of the measurement, it is assumed that the lengths measured using optical microscopy $\left(L_{1}, d_{1}\right.$, and $\left.d_{2}\right)$ are evenly distributed (uniform distribution) within the range of recorded values. The half-width of the measurement range is taken to be the length of 25 pixels (equivalent to $3 \mu \mathrm{m}$ at $50 \times$ ) in the digital imaging system; the probability of the actual value lying outside of this interval is small (Type B evaluation). The feature lengths were measured such that no visibly detectable damage was contained with the distances $d_{1}$ and $d_{2}$. The uncertainty in the flycutter radius $R$ is evaluated using a series of observations (Type A evaluation) with a normal distribution. Table 3 tabulates the uncertainty components in the critical chip thickness calculation. Fig. 11 shows sample cuts

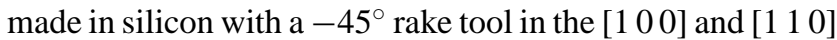
directions.

\section{Results}

A distinct crystallographic orientation dependence is found in the experimental results. The cutting forces, critical chip thickness, and machining damage vary in a cyclical pattern that repeats every $90^{\circ}$, just as the crystal structure repeats every $90^{\circ}$ on the cubic plane. The force data over two consecutive $90^{\circ}$ sections of the $\left(\begin{array}{lll}0 & 0 & 1\end{array}\right)$ workpiece are shown in Fig. 12 (1400 mm/s cutting velocity). The force data are normalized by the maximum chip area of each individual cut, effectively eliminating variations in force due to workpiece flatness. However, it must be kept in mind that these values are limited by the bandwidth of the measurement, which is fairly low with respect to the duration of the cut. This limitation effectively averages the actual cutting forces into a representative value for each crystallographic orientation in which the forces of both the ductile and brittle regimes are smeared together. As expected, the data show that the forces are generally higher in the more ductile [ 1100$]$ direction.

Fig. 13 shows the variation in critical chip thickness measured with $5600 \mathrm{~mm} / \mathrm{s}$ cutting velocity and $\mathrm{a}-45^{\circ}$ rake tool as a function of orientation. The calculated value for critical

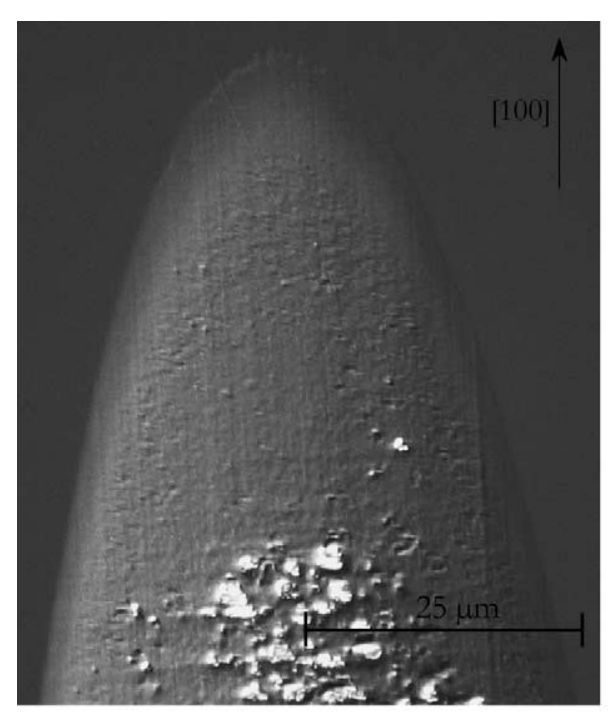

(a)

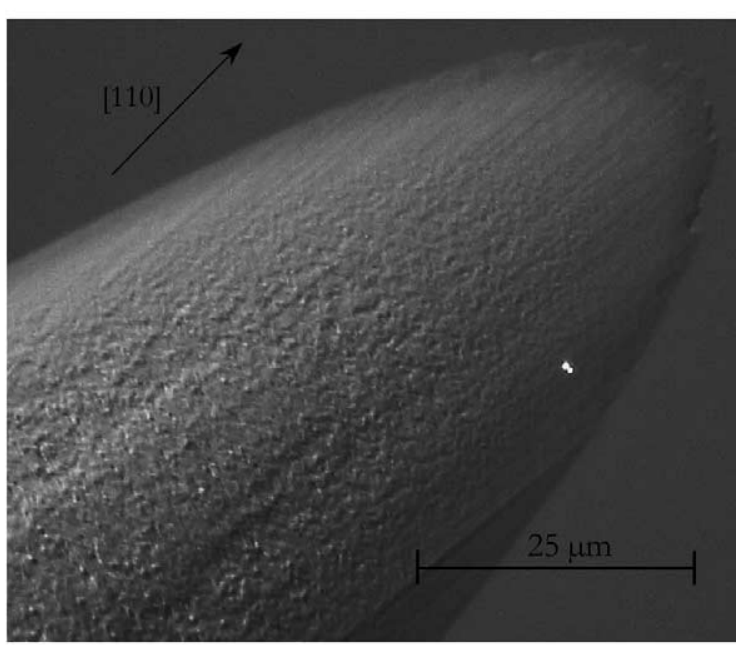

(b)

Fig. 11. Example of cuts made in a silicon workpiece with a $-45^{\circ}$ rake tool in the (a) [ 1000$]$ direction and (b) [ $\left[\begin{array}{lll}1 & 1 & 0\end{array}\right]$ direction ([0 001$]$ crystal plane). 


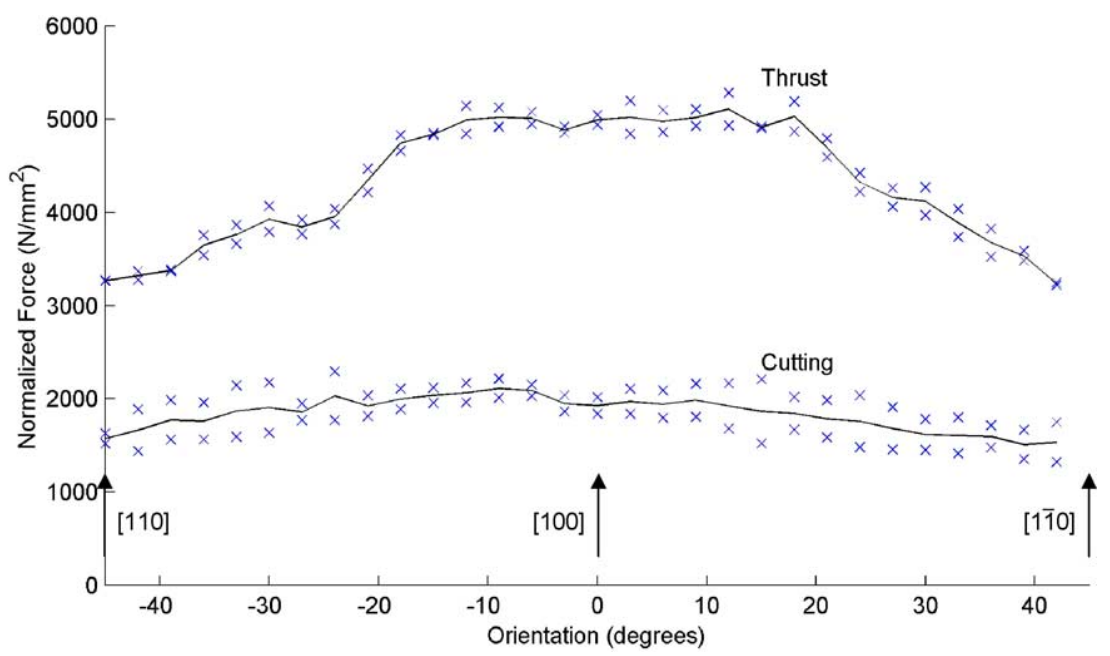

Fig. 12. Measured force data as a function of crystal orientation with a $-45^{\circ}$ rake angle tool, $(001)$ silicon, and $1400 \mathrm{~mm} / \mathrm{s}$ cutting velocity.

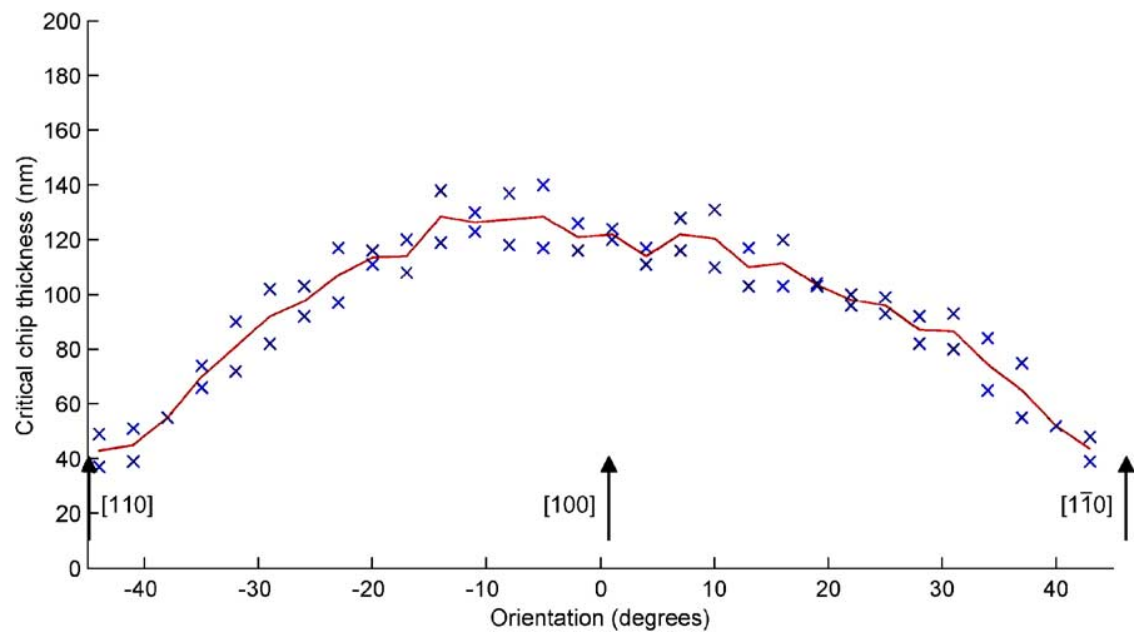

Fig. 13. Calculated critical chip thickness as a function of crystal orientation with a $-45^{\circ}$ rake angle tool $(001)$ silicon, and $5600 \mathrm{~mm} / \mathrm{s}$ cutting velocity.

chip thickness varies by as much as a factor of three with

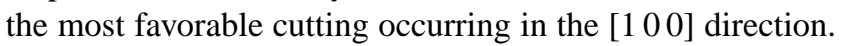
Although Wilks and Wilks do not provide directly comparable results for chemically similar diamond, their pioneering work shows similar trends in both polishing and grinding tests where the [1 000$]$ direction is much easier to machine than in the [llll 110$]$ direction [6].

\section{Conclusions}

In this work, silicon flycutting experiments were performed to quantify the critical chip thickness at which ductile material removal gives way to brittle fracture in non-overlapping cuts. Representative values were also measured for the machining force variation around the $\left(\begin{array}{lll}0 & 0 & 1\end{array}\right)$ cubic face of monocrystalline silicon. Using a $-45^{\circ}$ rake tool, the critical chip thickness varies by a factor of three between the easy [ 1000$]$ and hard [ 110 l 10 directions, which is consistent with qualitative work done by previous researchers. If a diamond turning operation were configured such that the critical chip thickness were somewhere between the [1 110$]$ direction limit of $40 \mathrm{~nm}$ and the [1 00 ] direction limit of $120 \mathrm{~nm}$, the familiar four-lobed star damage pattern would be plainly visible in the finished workpiece.

This critical chip thickness variation has major implications for the production of plano silicon components by single-point diamond machining. For example, if a plano optic is turned on a rotating spindle, the tool will explore the entire crystallographic structure in each revolution of the spindle. As a result, the maximum material removal rate is restricted by the critical chip thickness in the hard machining directions on a given plane of the crystal. If the same plano optic can be made by flycutting, then it should be oriented such that the spinning tool explores only a small arc of the 
crystal near the easy cutting [ 100$]$ direction to allow the largest material removal rate.

\section{Acknowledgments}

The authors would like to acknowledge the support of the several collaborators who made this work possible from Aerotech, Edge Technologies, Kistler Instrument, Lion Precision, Moore Tool, and Professional Instruments.

\section{References}

[1] Paul E, Evans C, Mangamelli A, McGlauflin M, Polvani R. Chemical aspects of tool wear in diamond turning. Prec Eng 1996;18(4):4-19.

[2] Syn C, Taylor J, Donaldson R, Shimada S. Ductile-brittle Transition of Cutting Behavior in Diamond Turning of Single Crystal Silicon. LLNL UCRL-98100, 1988.

[3] Bifano T, Dow T, Scattergood R. Ductile-regime grinding of brittle materials: experimental results and development of a model. In: Proceedings of the SPIE: Advances in Fabrication and Metrology for Optics and Large Optics, 1988;966:108-15.

[4] Blake P, Scattergood R. Ductile-regime machining of germanium and silicon. J Am Ceram Soc 1990;73(4):949-57.

[5] Blaedel K, Taylor J, Evans C. Ductile-Regime Grinding of Brittle Materials. In: Jahanmuir S, Ramulu M, Koshy P, editors. Machining of Ceramics and Composites. New York: Marcel Dekker, 1999. pp. 139-76.

[6] Wilks J, Wilks E. Properties and Applications of Diamond. Oxford: Butterworth-Heinemann, 1991.
[7] Blackley W, Scattergood R. Crystal orientation dependence of machining damage - a stress model. J Am Ceram Soc 1990;73(10):3113-5.

[8] Shibata T, Fujii S, Makino E, Ikeda M. Ductile-regime turning mechanism of single-crystal silicon. Prec Eng 1996;18(2/3):129-37.

[9] Domnich V, Gogotsi Y. Effect of phase transformations on the shape of the unloading curve in the nanoidentation of silicon. Appl Phys Lett 2000;76(16):2214-6.

[10] Vodenitcharovna T, Zhang L. A mechanics prediction of the behavior of monocrystalline silicon under nanoidentation. Int J Solids Struct 2003;40(12):2989-98.

[11] Patten J, Cherukuri H, Yan J. Ductile regime machining of semiconductors and ceramics. In: Gogotsi Y, Domnich Y, editors. High Pressure Surface Science and Engineering, 2004, IoP (Institute of Physics).

[12] Nye J. Physical Properties of Crystals. Oxford: Clarendon Press, 1957.

[13] Wonsiewicz B, Chin G. A theory of Knoop hardness anisotropy. In Science of Hardness Testing and its Applications, Chapter 12. Ohio: American Society of Metals, 1973.

[14] Brookes C, Burnand R. Hardness anisotropy in crystalline solids. In: Science of Hardness Testing and its Applications, Chapter 15. Ohio: American Society of Metals, 1973.

[15] Pharr G, Oliver W, Clarke D. The mechanical behavior of silicon during small-scale indentation. J Electron Mater 1990;19(9):881-7.

[16] Chen C, Leipold M. Fracture toughness of silicon. Am Ceram Soc Bull 1980;59(4):469-72.

[17] Preus W, Riemer O. Single Point Diamond Machining. ASPE Annual Meeting Tutorial, 2003

[18] Brinksmeier E, Preuss W, Riemer O, Malz R. Ductile to brittle transition investigated by plunge-cut experiments in monocrystalline silicon. In: Proceedings of the ASPE 1998 Spring Topical Meeting. 1998;17:55-8.

[19] ANSI/NCSL Z540-2, US Guide to the Expression of Uncertainty in Measurement, 1997. 